\title{
GPR Monitoring of Volumetric Water Content in Soils Applied to Highway Construction and Maintenance
}

\author{
K. Grote ${ }^{1}$, S. Hubbard ${ }^{2}$, Y. Rubin ${ }^{1}$
}

${ }^{1}$ Dept. of Civil and Env. Engineering, UC Berkeley, Berkeley, CA 94720 krgrote@,lbl.gov

${ }^{2}$ Lawrence Berkeley National Laboratory, Berkeley, CA 94720

\section{Introduction}

Detailed knowledge of the subsurface water content is important for highway design, maintenance, and repair. Transportation engineers can monitor the water content of sub-asphalt soils to estimate the soil stiffness as an index of the likely performance of a pavement and to evaluate the need for subsurface drainage retrofits. Conventional approaches for measuring water content include gravimetric sampling, time domain reflectometry [TDR], and neutron probes, all of which are time-consuming and invasive. Additionally, each of these methods provides only point measurements; since soil moisture content can vary greatly over space and time, point measurements are of limited value when surveying over a large area and over a period of time. An alternative to these conventional methods is ground penetrating radar (GPR), which can be used to quickly collect continuous, high-resolution water content estimates. GPR techniques can be used to estimate water content due to the sensitivity of electromagnetic velocity to water content. The two experiments described here, a controlled pit study and a transportation application in sub-asphalt soils, are based upon measuring the velocity of common-offset GPR reflections, which allows estimation of water content over deeper intervals than is possible with groundwave data and more quickly than can be accomplished with common-midpoint surveys. To estimate the velocity from GPR reflections, both the travel path and the travel time of the electromagnetic energy must be known. With commonoffset GPR techniques, the two-way travel time of the energy from the surface to the reflective interface and back is measured. To estimate electromagnetic velocity, the depth of the reflective interface must be determined. For engineered materials, the depth of a reflective layer might be known from construction records; for natural soils, calibration boreholes could be used to estimate the depth of soil layer interfaces.

After the electromagnetic velocity $(v)$ has been calculated, it can be converted to the real part of the dielectric constant, $\kappa$, using the following relationship: 


$$
\kappa=\left(\frac{c}{v}\right)^{2}
$$

where $c$ is the plane-wave propagation velocity of electromagnetic waves in free space (Davis and Annan, 1989). A petrophysical relationship can be used to convert $\kappa$ to volumetric water content $\left(\theta_{v}\right)$. One commonly used petrophysical relationship is a volumetric mixing model developed by Roth et al. (1990):

$$
\theta_{v}=\frac{\kappa_{\text {effective, mixing }}^{1 / 2}-(1-\eta) \kappa_{\text {solid }}^{1 / 2}-\eta \kappa_{\text {air }}^{1 / 2}}{\kappa_{\text {water }}^{1 / 2}-\kappa_{\text {air }}^{1 / 2}}
$$

where $\eta$ is porosity, $\kappa_{\text {air }}, \kappa_{\text {solid }}$, and $\kappa_{\text {water }}$ are the dielectric constants of the air, solid, and water components of the mixture, respectively, and $\kappa_{\text {effective,mixing }}$ is the estimate of dielectric constant obtained from electromagnetic measurements. For increased accuracy, site-specific relationships can also be developed.

\section{Water Content Estimation in Sandy Test Pits}

\subsection{Construction of Test Pits}

To determine the accuracy of common-offset GPR for providing estimates of water content, three test pits filled with sandy soil having carefully controlled water contents were constructed. During construction, reflectors were buried at measured depths throughout the pits. The first pit had sides $3 \mathrm{~m}$ in length, was $1.5 \mathrm{~m}$ deep, and had an average $\theta_{v}$ of 0.20 . The other two pits had sides $2 \mathrm{~m}$ in length and were $0.8 \mathrm{~m}$ deep; one of these pits had a $\theta_{v}$ of 0.06 and the other was 0.12 . Test pits were employed instead of natural soil deposits to enable control of the water content and to ensure homogeneity of the soil throughout the test volume. Under these conditions, the variations in $\kappa$ obtained from GPR reflection data could be investigated solely as a function of $\theta_{v}$. A well-sorted, silty sand was

chosen as the test soil, since this soil type is optimal for GPR signal penetration and is similar in composition to the sub-asphalt materials used in the transportation industry. The depths of the pits were chosen based on the requirements for water content measurements transportation applications, and the lateral dimensions were designed to allow several reflectors to be buried in the pit sufficiently far apart as to avoid reflection interference between buried reflectors and the walls of the pit.

The soil was placed in the pits and compacted in successive layers of 10 to $15 \mathrm{~cm}$ in thickness. After compacting each layer, two to four soil samples of approximately $600 \mathrm{~g}$ each were taken from different locations on 
the layer surface, and the gravimetric water content $\left(\theta_{g}\right)$ was calculated for each sample. Two density measurements were also taken after the compaction of each layer using a Portaprobe Model B ${ }^{\circledR}$ Mark II nuclear density gauge. For each soil sample, $\theta_{v}$ was calculated by multiplying $\theta_{g}$ by the soil density. These measurements showed that $\theta_{v}$ did not vary laterally across each layer in any of the pits, though these values did vary somewhat vertically from one layer to another in the pit with $0.20 \theta_{v}$. Figure 1 shows the calculated profile of $\theta_{v}$ in each pit from these data. In addition to these measurements, neutron probe access tubes were also installed in the pits to monitor the distribution of $\theta_{v}$ with time.

Several different types of reflectors were buried in the test pits to determine which produced the clearest and most easily interpretable reflections. The buried reflectors included solid aluminum plates $(25 \mathrm{~cm}$ on each side and $1 \mathrm{~cm}$ thick), segments of hollow steel and aluminum pipes $(21 \mathrm{~cm}$ long, $11 \mathrm{~cm}$ in diameter, with walls $0.5 \mathrm{~cm}$ thick), and hollow PVC plates filled with water (PVC sheets $18 \mathrm{~cm}$ on each side and $0.25 \mathrm{~cm}$ thick, with $2 \mathrm{~cm}$ of water between the upper and lower sheets). The reflectors were buried during layer compaction at depths ranging from 11 to $120 \mathrm{~cm}$ below the pit surface, and different configurations of reflectors were tried. Most reflectors were simply buried in the pit, but some reflectors were 'stacked', or buried over another reflector, with a vertical distance of at least $18 \mathrm{~cm}$ between each reflector. The locations of the reflectors and the access tubes for the pit with $0.20 \theta_{v}$ are shown in map view in Figure 2. The location and elevation of each reflector were accurately surveyed during construction of the pits.

\subsection{Data Collection}

After the test pits were completed, geophysical surveys were performed using surface GPR and neutron probe measurements. The GPR surveys were collected along several traverses across each pit; each traverse passed over two or more of the buried reflectors, as illustrated in Figure 2. GPR measurements were taken using four central frequencies: $225,450,900$, and $1200 \mathrm{MHz}$, with a $1 \mathrm{~cm}$ station spacing for all frequencies. The GPR surveys showed very clear responses from the buried reflectors at all frequencies; the reflections arrived in a hyperbolic pattern as the GPR apparatus was pulled over the reflectors. Figure 3 shows reflections collected at a central frequency of $450 \mathrm{MHz}$ in the pit with $0.20 \theta_{v}$ from two aluminum plate reflectors buried at depths of $0.60 \mathrm{~m}$ and $0.85 \mathrm{~m}$ below ground surface [BGS]. 
Neutron probe data were collected at a downhole sampling interval of $5 \mathrm{~cm}$ coincidentally with the surface GPR surveys. Neutron probe data are measured in "backscattered counts", which can be related to water content. The neutron probe measurements in each pit were very similar in all of the access tubes at each depth, which supports the lateral uniformity of water content shown in the $\theta_{g}$ measurements (Figure 1).

After the geophysical measurements were completed, the pits were excavated, and additional $\theta_{g}$ measurements were taken at various depths. These measurements were compared to those taken during pit construction. From the similarity of the $\theta_{g}$ measurements before and after the geophysical surveys, the $\theta$ distribution in each pit was determined to be constant with time.

\subsection{Estimation of Water Content from Surface GPR Measurements}

The $\theta_{v}$ in each pit was estimated by calculating the electromagnetic velocity between the surface and the buried reflectors and also between the 'stacked' buried reflectors. The velocity from the surface to the buried reflectors was calculated by measuring the travel time from the air wave, corrected for onset delay, to the apex of the reflection hyperbola. This travel time is the interval in which energy travels vertically from the ground surface to the buried reflector and back, and it occurs when the GPR apparatus is directly over the center of the reflector. Using this travel time ( $\Delta \mathrm{t}_{0}$ in Figure 3$)$ and the known depth to the reflector, the velocity can be calculated, and $\kappa$ can be determined using equation (1). This $\kappa$ is an average value for the vertical interval between the surface and the buried plate. The velocity over the interval between stacked reflectors was calculated in a similar manner, by taking the difference in travel times between the two hyperbola peaks. This method reduces the uncertainty inherent in picking the absolute 'zero time' from the air wave and allows calculation of $\kappa$ over smaller vertical intervals.

After $\kappa$ was determined for each depth interval, both soil-specific and published petrophysical relationships were used to estimate $\theta_{v}$. The soil-specific relationship was developed using laboratory measurements of $\kappa$ collected with a Trase Model $6050 X 1$ TDR apparatus with $15 \mathrm{~cm}$ waveguides and corresponding $\theta_{v}$ measurements. By fitting a curve through this data, the following soil-specific petrophysical relationship was created:

$$
\theta_{v}=-0.0006 \kappa^{2}+0.294 \kappa-0.092 \text {. }
$$


Figure 4 shows the comparison of the $\theta_{v}$ estimates derived from GPR data using equations (2) and (3) with the $\theta_{v}$ measurements obtained gravimetrically. The gravimetrically-derived $\theta_{v}$ values were arithmetically averaged over the vertical intervals in which the electromagnetic velocity was measured for comparison with the GPR estimates. Two GPR-obtained estimates are shown for each gravimetrically-obtained $\theta_{v}$ measurement; each GPR estimate is from a different traverse, and two traverses were taken over each reflector. Using the volumetric mixing model (equation (2)) with a measured average porosity of 0.26 and $\kappa_{\text {solid }}$ of 6.3 , the average absolute difference between the measured and estimated $\theta_{v}$ in the test pits was less than 0.012. Using the soil-specific petrophysical relationship (equation (3)), the average absolute difference was less than 0.008 .

The accuracy of the GPR estimates did not appear to be influenced by $\theta_{v}$, but it was affected by the geometry of the buried reflectors. The 'stacked' reflectors gave the most accurate $\theta_{v}$ estimates, while the estimates over the intervals from the ground surface to a reflector were slightly less accurate. This result is likely due to uncertainty in the exact time that the GPR signal enters the ground; this inaccuracy does not affect betweenreflector estimates. All reflector types gave clear reflections, but certain configurations were easier to interpret. The stacked reflectors were most efficient if the overlying reflector was thin (e.g., an aluminum plate) and did not generate reflections from both the top and bottom of the reflector. Reflectors that were placed at least $20 \mathrm{~cm}$ below the ground surface were also easier to interpret, as the ground wave did not interfere with reflected arrivals at this depth.

In addition to the reflector geometry, errors in the GPR estimates of $\theta_{v}$ could also be produced from uncertainties associated with picking the air and reflected waves, digitization of the GPR data, the petrophysical relationship invoked, and the depth measurement of the reflector. Although error due to picking the air and reflected waves is difficult to quantify, error analysis was performed to investigate the influence of the other factors. This analysis showed that the greatest cause for error was inaccurate depth measurements, with shallow reflectors having proportionately higher errors. The maximum and minimum $\theta_{v}$ errors predicted were 0.048 and 0.003, which corresponded to the shallowest and deepest reflectors, respectively. Comparison of the GPR-obtained estimates of $\theta_{v}$ with the gravimetrically-obtained measurements (Figure 4) illustrates that the GPR estimates are within the bounds suggested by error propagation analysis, and the measured error is usually considerably less than 
that predicted by this analysis. Increased error is observed at the shallowest reflectors, which demonstrates that the intervals over which $\theta_{v}$ are estimated must be sufficiently thick so that small errors in depth estimation do not cause large errors in estimating $\theta_{v}$.

\section{Water Content Estimation in Sub-asphalt Soils}

Based upon the success of using common-offset GPR for $\theta_{v}$ estimation in the sandy test pits, we applied this technique to the engineering application of monitoring $\theta_{v}$ in sub-asphalt aggregate soils. In this experiment, GPR surveys were collected in a grid over two differently designed pavement sections. The sections differed by the composition and thickness of the sub-asphalt aggregate layers and by the presence of a drainage layer in one of the sections. For both sections, water was injected into the aggregate layers, and additional GPR time-lapse surveys were collected over a period of several months. Finally, both sections were subjected to dynamic loading to simulate trafficking, followed by GPR data acquisition. Analysis of the GPR signal travel time through the aggregate layers prior and subsequent to both infiltration and loading allows estimation of the changes in $\theta_{v}$ with space and time due to natural drainage and dynamic loading. This information can be used to compare differently designed pavements and to correlate $\theta_{v}$ estimates with traditional pavement strength measurements. The following discussion, which is a summary of a single experiment conducted in one of the pavement sections prior to loading, demonstrates a practical application of GPR technology for obtaining $\theta_{v}$ estimates.

\subsection{Site Description and Data Collection}

The pavement section for this experiment was constructed by a contractor according to typical California Department of Transportation standards. The pavement was composed of four layers: asphalt rubber overlay, asphalt concrete (AC), aggregate base (AB), and aggregate sub-base (ASB), as shown in Figure 5. The rubber overlay and asphalt concrete have very low permeability and do not accumulate a significant amount of water, so this experiment focused on monitoring $\theta_{v}$ in the aggregate layers. The upper aggregate layer, the $\mathrm{AB}$, is heavily compacted and is composed of medium to coarse gravel in a matrix of silt and clay; the fines account for approximately $10 \%$ (by weight) of the $\mathrm{AB}$. The $\mathrm{ASB}$ is similar to the $\mathrm{AB}$ in composition, but has a slightly higher percentage of fines and is not compacted. The ASB is the lowermost engineered layer; the soil below it is the subgrade (SG), which at this site is a highly plastic deltaic clay. The pavement section is located inside a hangar, 
so the influences of the outside environment on $\theta_{v}$ should be negligible.

Water was introduced into the sub-asphalt aggregate layers by means of a drip infiltration system. Infiltration holes were drilled upgradient of the pavement test section through the rubber overlay and asphalt concrete at $0.5 \mathrm{~m}$ intervals for a length of $12 \mathrm{~m}$; the infiltration holes are shown as red circles in Figure 6 . Water was injected into these holes four times a day for a total injection of 3 gal/day over the $12 \mathrm{~m}$ zone; injection continued for a period of eight months. GPR data were collected over the test pavement at central frequencies of 900 and $1200 \mathrm{MHz}$ prior to infiltration and periodically during the infiltration period. GPR traverses were collected using a $2 \mathrm{~cm}$ station spacing along the grid indicated by blue arrows in Figure 6 . The data collected at $1200 \mathrm{MHz}$ along one GPR traverse is shown adjacent to the pavement cross-section in Figure 5. After the infiltration experiment was completed, eight $15 \mathrm{~cm}$ boreholes were drilled, and samples of $\theta_{g}$ were collected over the full thickness of the aggregate layers. These samples were converted to $\theta_{v}$ using the density of the aggregate layers given in construction records for subsequent comparison with the GPR-obtained estimates.

\subsection{Estimates of Water Content in Sub-asphalt Layers}

The $\theta_{v}$ in the sub-asphalt aggregate layers was estimated by measuring the travel time of the GPR signal through each layer, then calculating the velocity using the travel time and the known thickness of each layer. The velocity was converted to $\theta_{v}$ using a soil-specific petrophysical relationship developed in the laboratory using TDR. The estimates of $\theta_{v}$ obtained using this technique were compared to those obtained from gravimetric sampling and were found to agree well: the $\theta_{v}$ estimates from GPR data varied from those obtained gravimetrically by a maximum of 0.025 in the $\mathrm{AB}$ and 0.027 in the ASB, and the average absolute difference between the GPR estimates and gravimetric measurements of $\theta_{v}$ for the AB and ASB were 0.008 and 0.011 , respectively. One cause of error in the GPR-obtained estimates of $\theta_{v}$ was variations in the thicknesses of the aggregate layers that were not accounted for in the velocity calculations. Even with these errors, the GPR estimates of $\theta_{v}$ were accurate enough to be useful for pavement applications. The $1200 \mathrm{MHz}$ GPR data had the highest resolution and gave the best correlation with gravimetrically-obtained $\theta_{v}$ measurements $(\mathrm{R}=0.83)$; the results presented here are from the 1200 MHz data.

Using estimates of $\theta_{v}$ obtained from the GPR survey grid, the $\theta_{v}$ distribution in the sub-asphalt aggregate 
layers was estimated over space and time. By observing changes in the $\theta_{v}$ distribution with time, some of the hydrological properties of the aggregates can also be estimated. One method of observing changes in $\theta_{v}$ is to calculate the change in $\theta_{v}$ at each point between the post-infiltration survey and the pre-infiltration (dry) survey. Figure 6 shows the changes in $\theta_{v}$ for the AB and ASB at 119 and 205 days after infiltration began. Negative changes indicate drying and are shown by yellow areas, while positive changes indicate water accumulation and are shown as blue areas. By identifying zones of wetting and drying in both aggregate layers, both vertical and horizontal flow paths can be inferred. In Figure 6, most of the water accumulation occurs in the ASB below the infiltration zone; this indicates that water may be flowing through vertically-inclined channels or flow paths in the lower permeability (higher compaction) $\mathrm{AB}$ and may be flowing more horizontally or being stored in the more permeable ASB. Localized areas of drying in the AB underlain by wetter zones in the ASB also support this hypothesis.

\section{Conclusions}

The experiments in the test pits and the pavement section show that common-offset GPR reflection data can be used to estimate $\theta_{v}$ to a high degree of accuracy. The methodology developed in these two experiments provides a technique for obtaining quick, non-invasive, accurate, and high-resolution estimates of $\theta_{v}$. This method is applicable at sites where the layer thickness (or depth to the reflector) can be estimated with sufficient accuracy and in areas where GPR performs well, such as in low loss materials. This common-offset GPR technique could provide a valuable addition to current $\theta_{v}$ estimation techniques under both engineered and natural conditions, and could be applied to various other subsurface applications in agriculture, ecology, and hydrology.

Acknowledgements: This study was funded by a grant from Caltrans, USDA 2001-35102-09866, and NSF EAR0087802 to Yoram Rubin. We sincerely thank John Harvey and Michael Riemer for their unfailing support and advice throughout the experiments, and Ed Diaz and staff for their assistance with data collection.

\section{References}

Davis, J.L. and Annan, A.P., Ground penetrating radar for high-resolution mapping of soil and rock stratigraphy, Geophysical Prosp., 27, 531-551, 1989.

Roth, K., Schlin, R., Fluhler, H., and Attinger, W., Calibration of time domain reflectometry for water content 
measurements using a composite dielectric approach, Water Resour. Res., 26(10), 2267-2273, 1990.

\section{Figure Captions}

Figure 1: Measured water content with depth in the three test pits. Two or more measurements were collected at different horizontal locations for each depth.

Figure 2: Map view of the pit with $0.20 \theta_{\nu}$ showing the surface location of the buried reflectors, surface GPR lines, and neutron probe access tubes. The reflectors in this pit were aluminum plates, each buried at a different depth.

Figure 3: Common-offset data collected over the pit with $0.20 \theta_{v}$, along the traverse labeled "Line 2" on Figure 2.

Figure 4: Comparison of GPR-derived estimates of $\theta_{v}$ with $\theta_{v}$ measurements obtained gravimetrically from all three test pits. Equations (2) and (3) produced estimates very similar to the gravimetric measurements, suggesting that common-offset GPR can be used as a reliable water content estimation tool.

Figure 5: Vertical cross-section of the test pavement illustrating the thickness of the rubber overlay, asphalt concrete (AC), aggregate base (AB), aggregate sub-base (ASB), and subgrade (SG) and a $1200 \mathrm{MHz}$ commonoffset GPR survey line over this pavement showing the interpreted reflections from each interface. The water infiltration hole penetrates through the rubber overlay and $\mathrm{AC}$ and allows water to drip directly onto the $\mathrm{AB}$.

Figure 6: Maps of the changes in water content observed at days 119 and 205 relative to pre-infiltration conditions in the $\mathrm{AB}$ and $\mathrm{ASB}$. The contour maps are kriged interpolations from data collected along the surface GPR traverses shown as blue arrows. The blue and yellow areas on the contour maps represent zones of wetting and drying, respectively. 


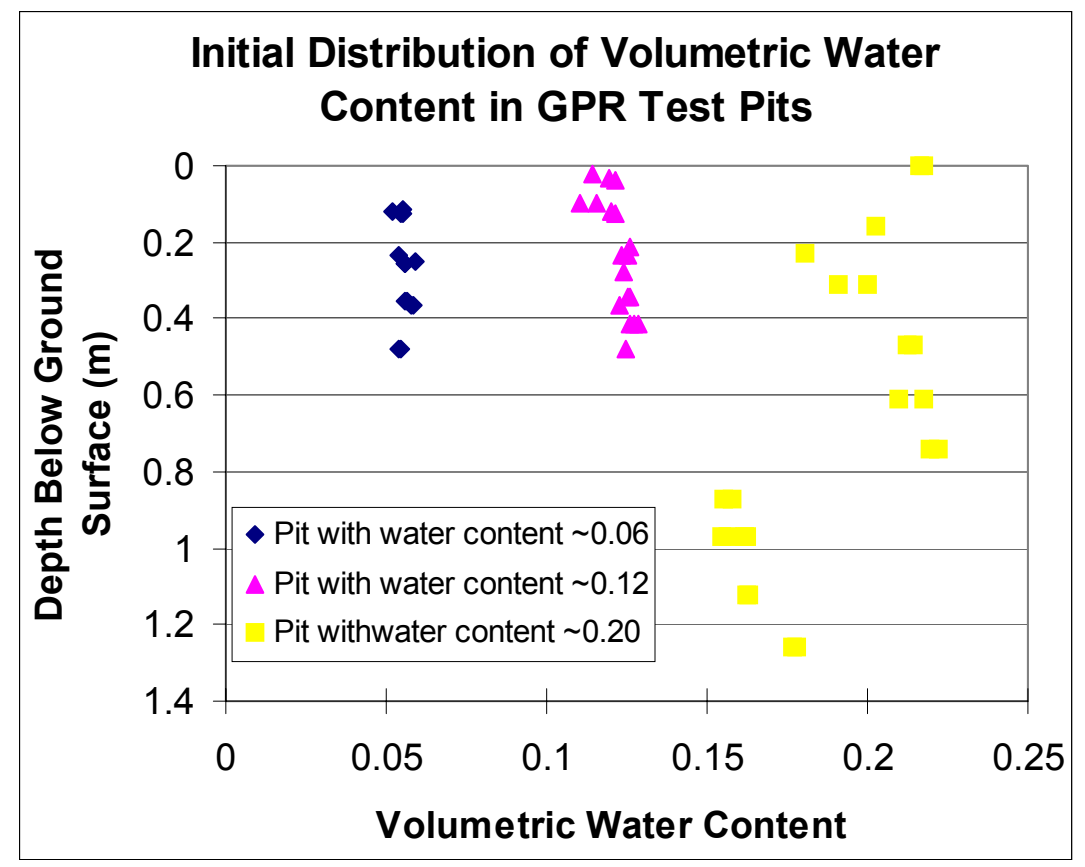

Figure 1

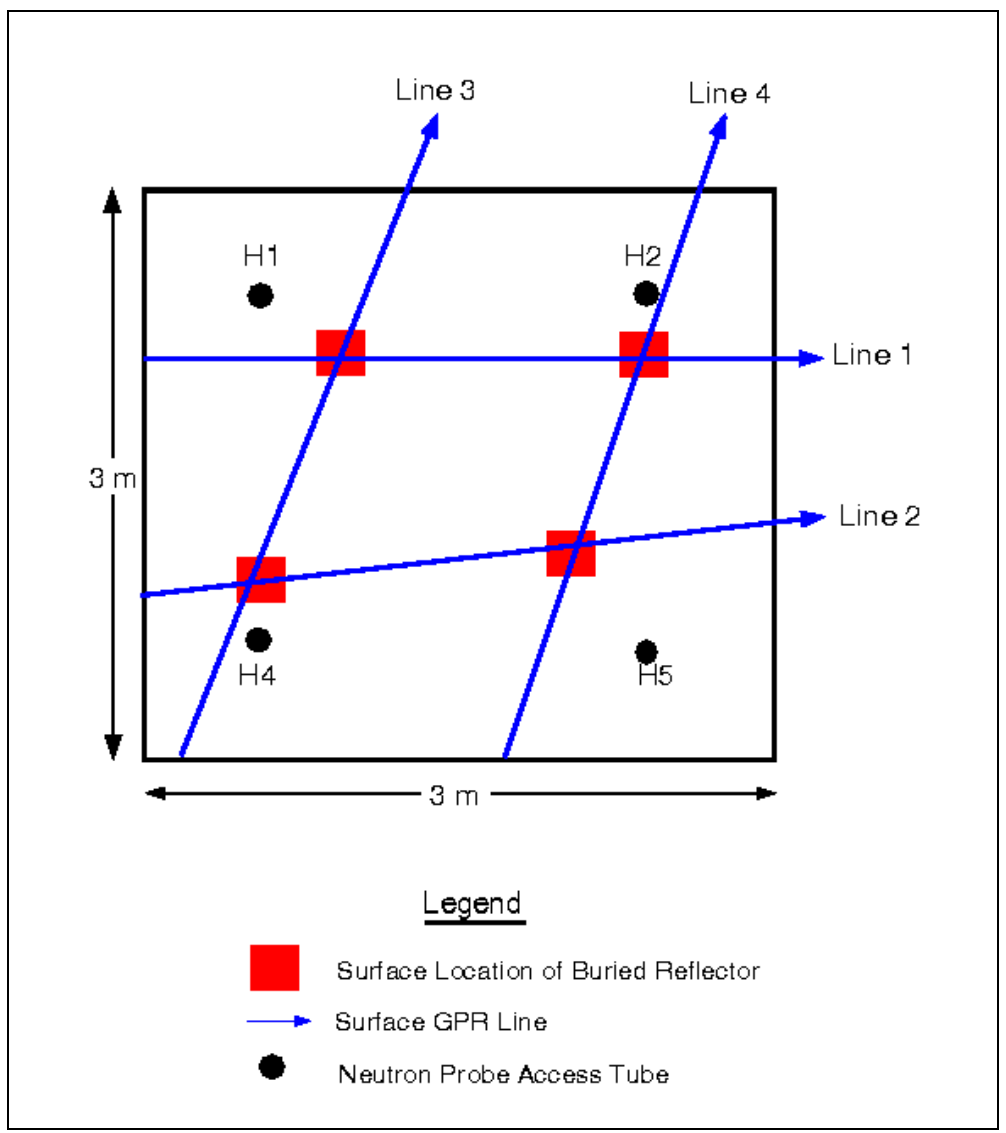

Figure 2 


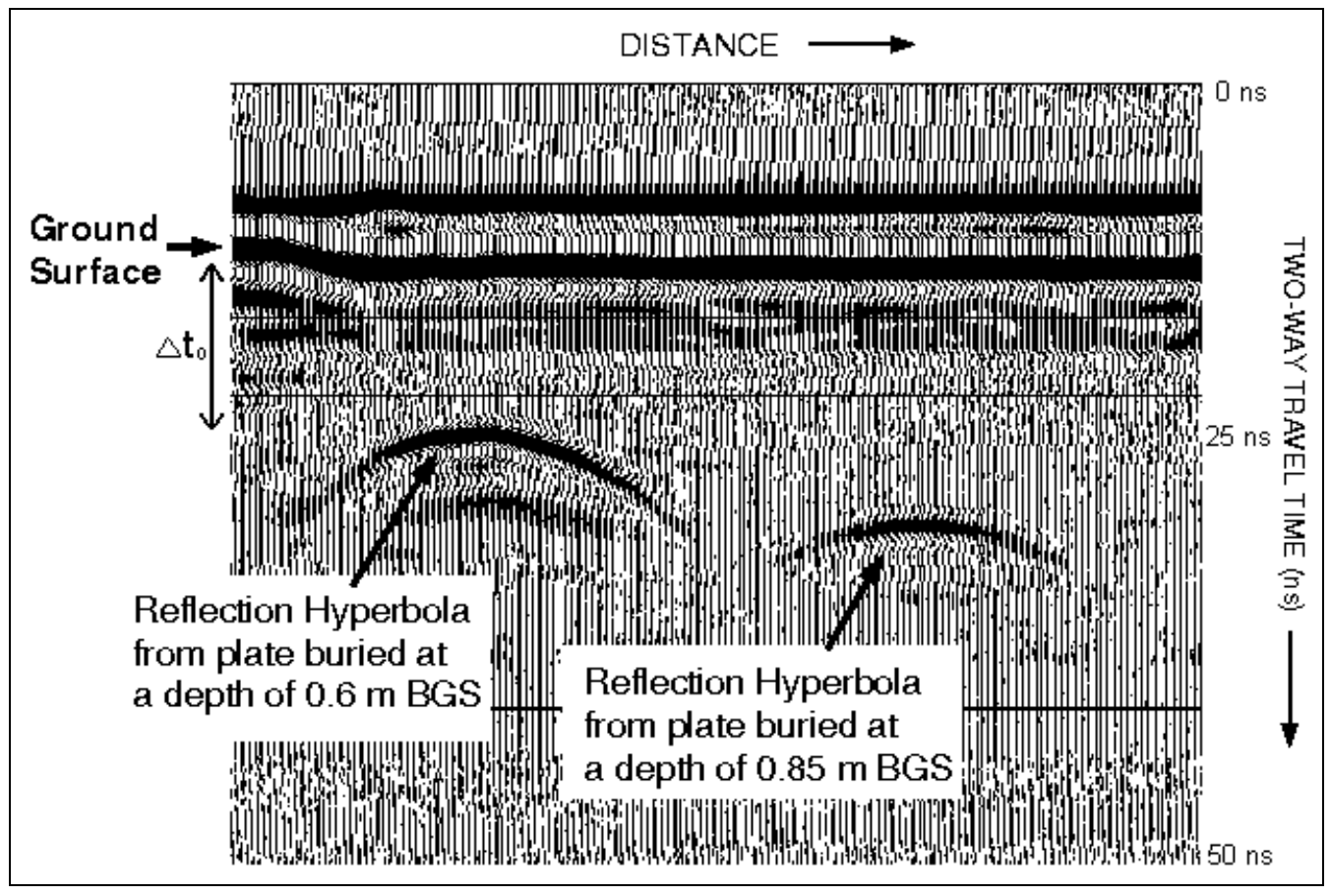

Figure 3

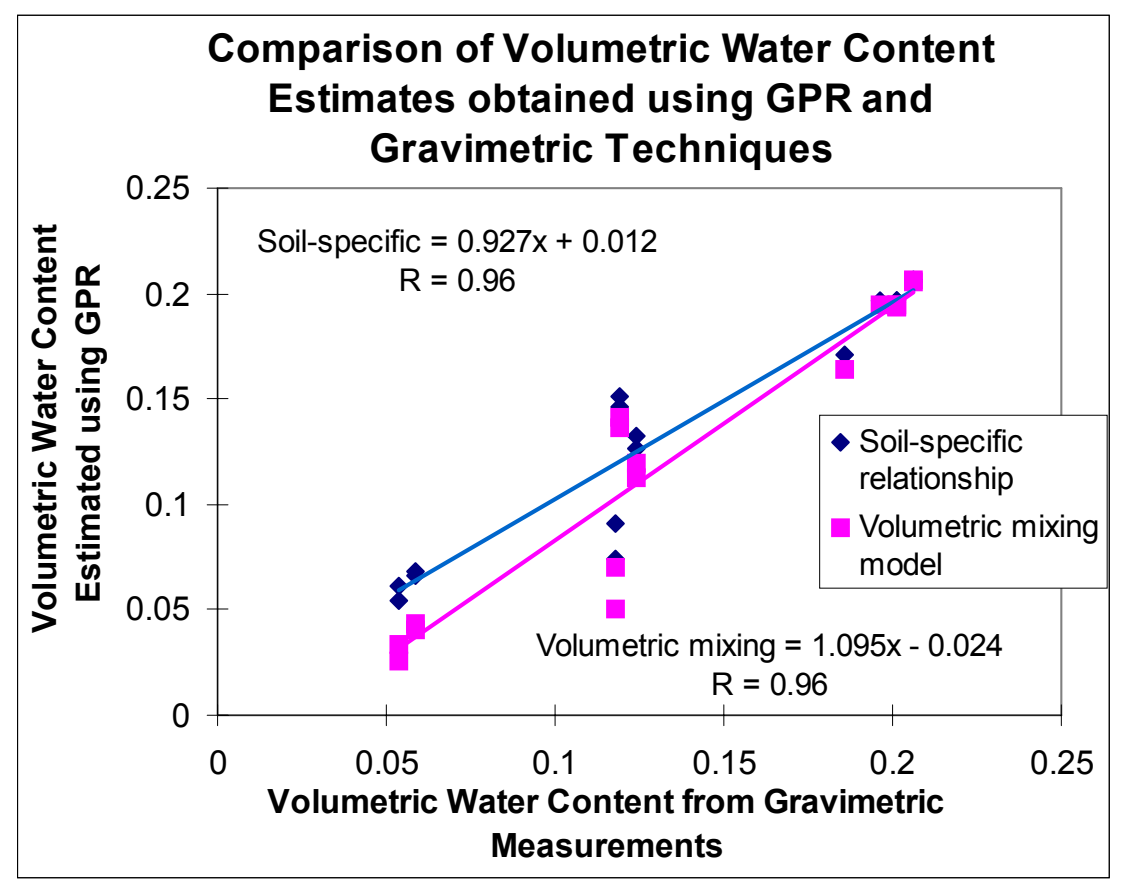

Figure 4 


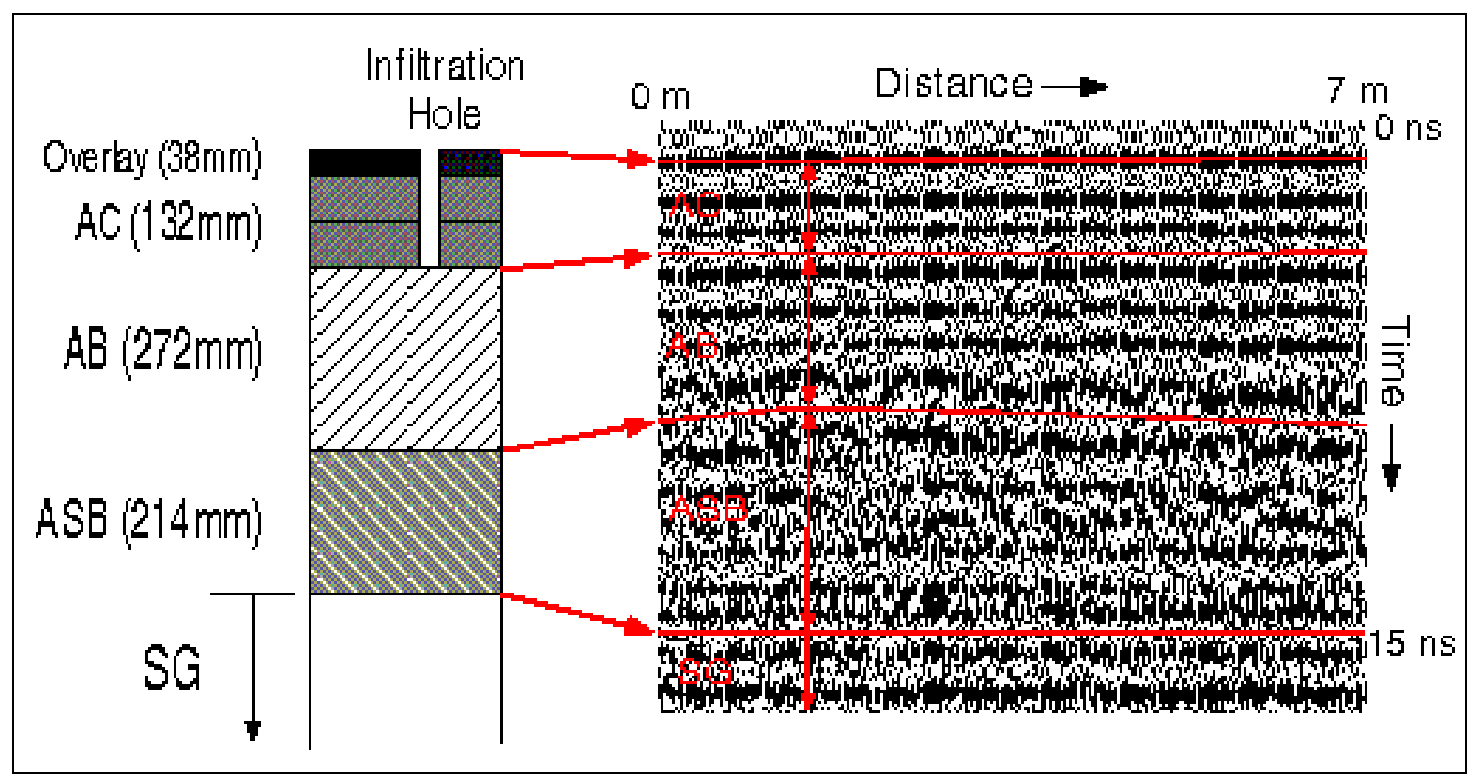

Figure 5 

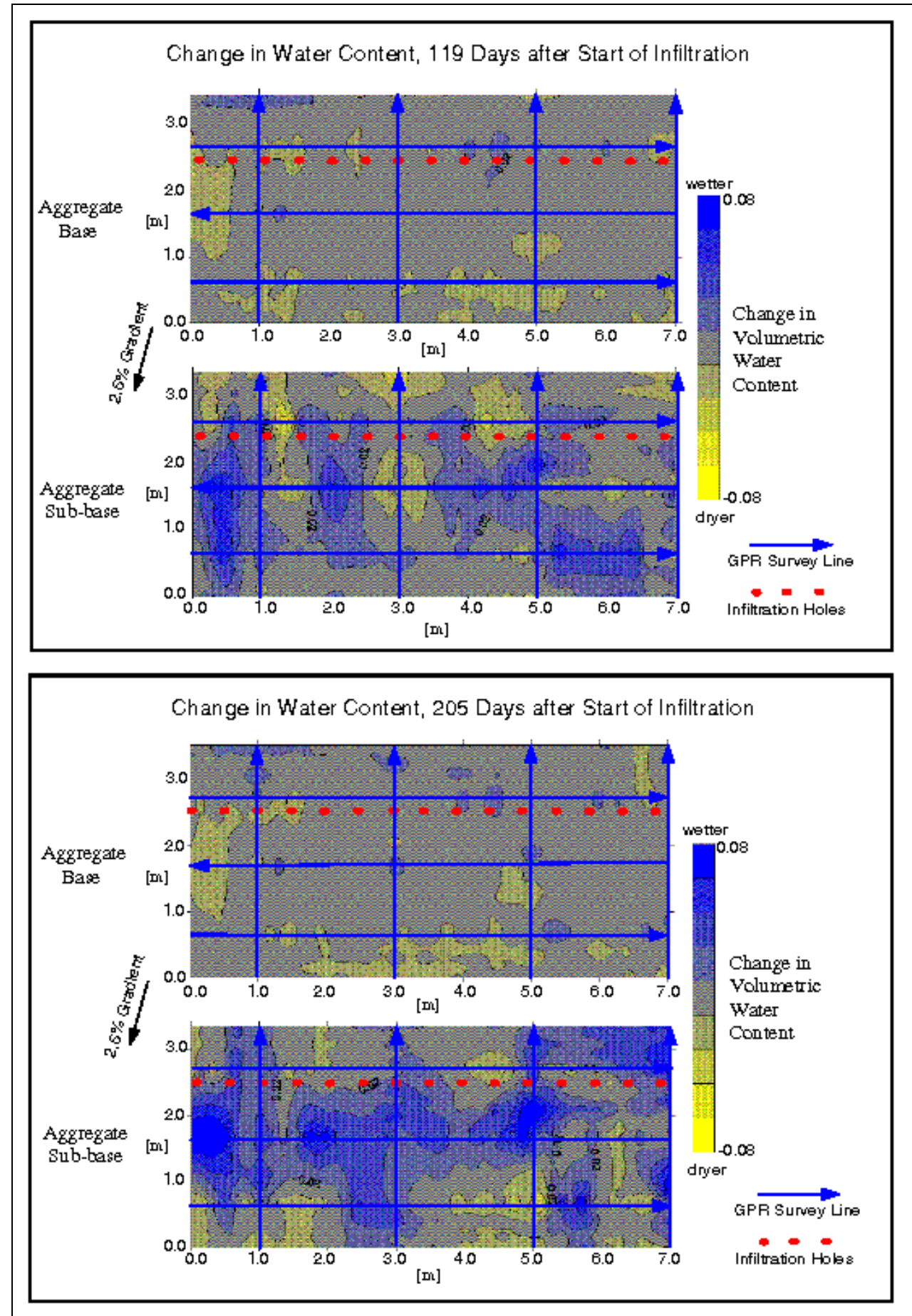

Figure 6 